\title{
DOCUMENTOS
}

\section{Cartas Inéditas de Amado Nervo, Rubén Darío y Enrique Gómez Carrillo}

Las siguientes cartas pertenecieron al doctor Javier Mojarrieta y su señora, de La Habana, Cuba, primos míos; como se puede ver, la correspondencia es el fruto de una amistad personal que existió entre los conocidos autores y los esposos Mojarrieta.

Mojarrieta estableció residencias en París y Madrid a'l mismo tiempo que Nervo, Darío y Gómez Carrillo vivieron en esas ciudades; las cartas indican que los autores fueron invitados especiales en la casa del médico cubano.

Durante mi última visita a Cuba, cuando la revolución cubana se había convertido en una realidad hasta el punto que tuve que ser evacuada, el hijo del matrimonio Mojarrieta me presentó estas cartas informándome que no eran más que un pequeño número de una colección muy abundante que empezó a fragmentarse años atrás cuando la señora de Mojarrieta regaló una cantidad de ellas a la Biblioteca Nacional de La Habana.

Las cartas que aquí presento podrían haber sido muchas más; las circunstancias del momento revolucionario cubano son las responsables de que muchas cartas, notas, etc., escritas por estos hombres hayan quedado olvidadas en la casa de los Mojarrieta en Cuba.
University of Illinois,
F. J. TORT
Cbicago Circle. 
Sci casa:

Paris, 5I, Rue de Miromesnil

3 de marzo

E L L I B E R A L

Marqués de Cubas, 7, Madrid

Sr. Lic. Spíndola

México

Mi muy distinguido compañero,

Aprovechando la hospitalidad tan amplia que siempre han encontrado mis prosas en El Mundo, envío hoy a $\mathrm{Ud}$. una silueta que forma parte de un libro que voy a publicar con el título de Los bispanoamericanos en Paris y que le ruego anuncie.

Dentro de poco podré enviar a Ud. periódicamente a'lgunas crónicas parisienses.

Con mi agradecimiento reciba Ud. la seguridad de mi consideración.

(F) Gómez Carrillo

P.S.-Le ruego me envíe Ud. el $\mathrm{N}^{\circ}$ que publique mi silueta esta. 
secoses:

EL LIBERAL

Marques de cubas, 7 , Madrid

PARIS, SI, RUE OE MIROMESNIL

3 de wese 0

S. Lie. Ipundola.

unéxico.

clli uncey distuiguido Conefuriero, Oprovachandos la Ros firtalidorol. tru aruplia que uanper tow encontrado nuis prozan en El Ulundo, envio lony à hol. nua silueta que forma part. de un libro que noy à fublican con al litulo de Los hispano amecrica.

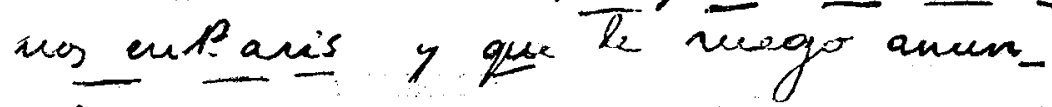
cie.

Dentro de foro podré en vion a ted periodicanmente algunas rónicas farisienses.

Con mi agradecimient recito has. la seguludeal de mis comsudenacioin,

Gomes canillo

P.S. - Le unego une anvig nd. Al nu que perblique mu silueta sta. 
Querido amigo,

Estoy escribiendo el artículo. Comeré aquí y a las $8 \mathrm{r} / 2$ iré a leérselo, para que se pueda ir por el correo del jueves.

Afectuosamente,

\section{(F) Gómez Carrillo}

P.S.-Si el Zulú está allí dígale que me hable a las 7 de la noche por el teléfono.

Madrid, marzo 3I - I900 
Lenesids annigo, Estoy escuibievds el articulo. Concuí aqui Y à las $81 / 2$ iré à learsel. frama qu se vueda. is." for el corres dal jucures. afrectios saluente,

Gounz Camilto

P. S. - Si el Zulí estáallí digalequ ue hable à las it de la woch for el talaforis.. 
Madrid, marzo 3I - I900

Mi querido amigo:

Mil gracias por los amables conceptos de su carta; y crea que mi amistad para U. ha sido en la ausencia, la afectuosa y sincera de siempre. Tanto a U. como al amigo Talero les he recordado por estos mundos.

Le felicito por el completo triunfo de que me habla. No podia ser de otro modo, dados su actividad y su inteligencia. En cuanto a su galante invitación de "Grand Seigneur" desde luego la acepto, agradeciéndosela muy cordialmente.

No he recibido aún orden de partir a Italia o Francia, por lo tanto, creo estar aquí a fines de Mayo todavía. Mi dirección, pues, será la misma en esta capital, Calle Marqués de Santa Ana, 29.

Aquí tengo un amigo cubano, el conde de O'Kelly; y ví también a Pichardo, el del Fígaro.

Hasta pronto, pues, mi querido amigo. Créame tal, y mándeme en lo que guste, como a su afmo.

(F) Rubén Darío 
Marid, Marro31-1900

Si quesiso aungo,

Mil gracias for Los ans. ber conception de su carta iy crea

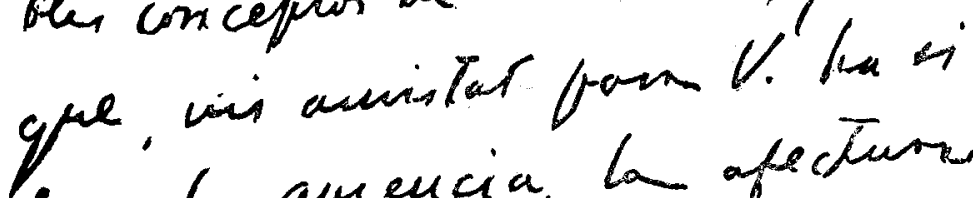
do enle amencia, la ofpetionos $y$ suncers de siecufpre. Fauto \& V. coner al amijo Zadero lei he recsitato fur citos nussose Le plicito por el conffetotriva to de gre we babla. No protion 


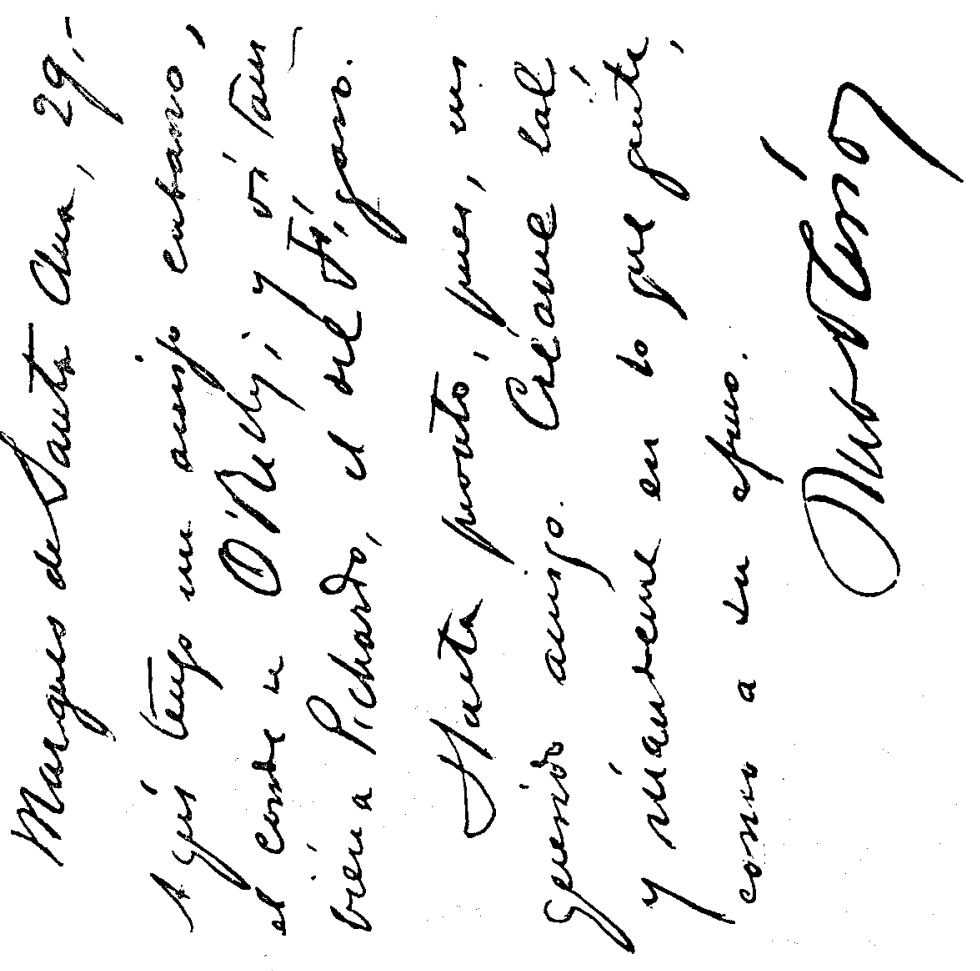

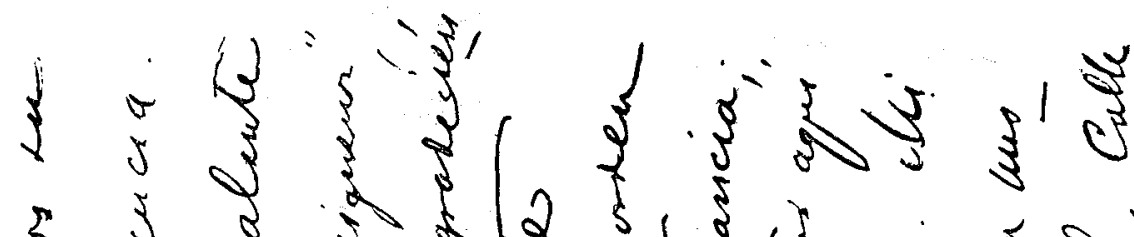

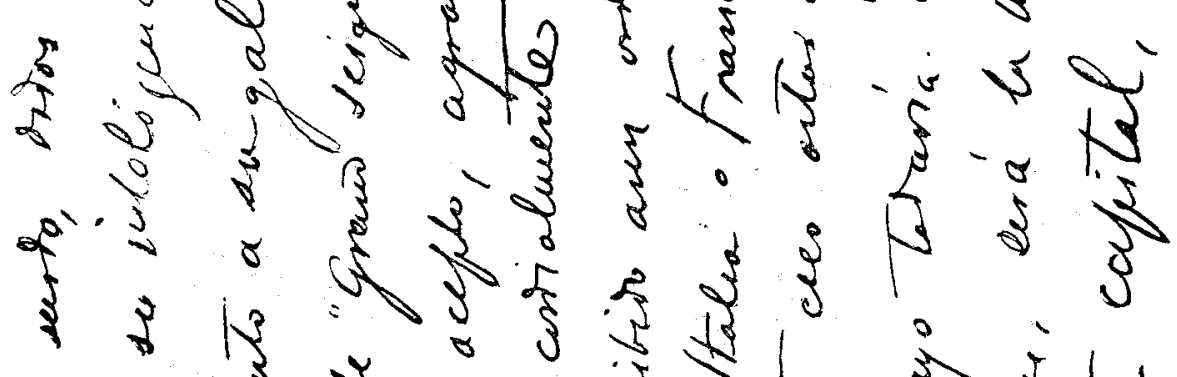

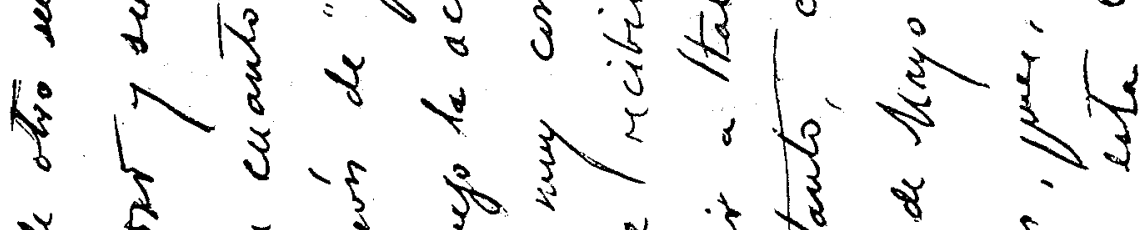

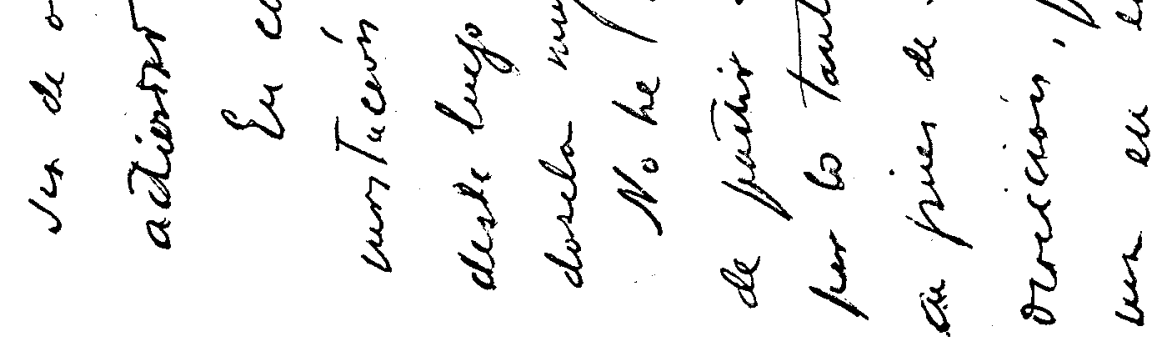


Jueves -26 -

Mi distinguido amigo:

Mañana en la noche tendrá $U$. en su casa el trabajo que me encarga, y que haré con mucho gusto. Le felicito por el constante progreso del éxito de su negocio.

Le saludo afectuosamente,

\author{
(F) Rubén Darío
}

París, Fbro., 25/90I 
Theres - 26.

mi sitimguiso amigo.

Mañana en

in nocke tenoral fo en in cass Itrataj's que me encarga, y gue ha ré con mucho punto. Le felicito for al contrante propeso gel $\ddot{x}_{-}$ to de ta regacio.

Letalus afeitars amente.

Ousér bariós 
Paris, Fbro. 25/90I

Muy querido amigo:

Me supongo que recibió mi carta anterior en la que le decía que vivía con Rubén Darío, 29, rue du Faubourg Montmartre y le daba las gracias por sus finezas. Una fineza más viene ahora a obligarme sobremanera, su fructífera recomendación en la Discusión. Figúrese que no hace ni tres dias, escribía al Cónsul de México Clemente Vázquez, pidiéndole que me recomendase con algún periódico de La Habana, pues hace tiempo que mi estancia en París es el problema de la trisección de los ánguilos o algo tan difícil como eso, y he aquí que usted - siempre oportuno- viene en mi ayuda. ;Gracias! Hoy escribo al Lic. Coronado remitiéndole mi primera carta y procuraré como U. me lo indica vincularme al periódico, lo cual deseo de todas veras.

Comprendo en todo su valor lo que hace por mí y me será sumamente grato hallar una forma de agradecerle. Usted sabe, cuán adicto le soy.

Ignoro el tiempo que Rubén y yo viviremos juntos, pero yo le avisaré de todas suertes mis direcciones sucesivas en esta peregrinación un poco agitada por París.

Mañana en paquete postal le dirijo El Bacbiller, traducido al francés.

Sabe que le quiere mucho y le está muy obligado.

Su amigo afectísimo

(F) Amado Nervo 


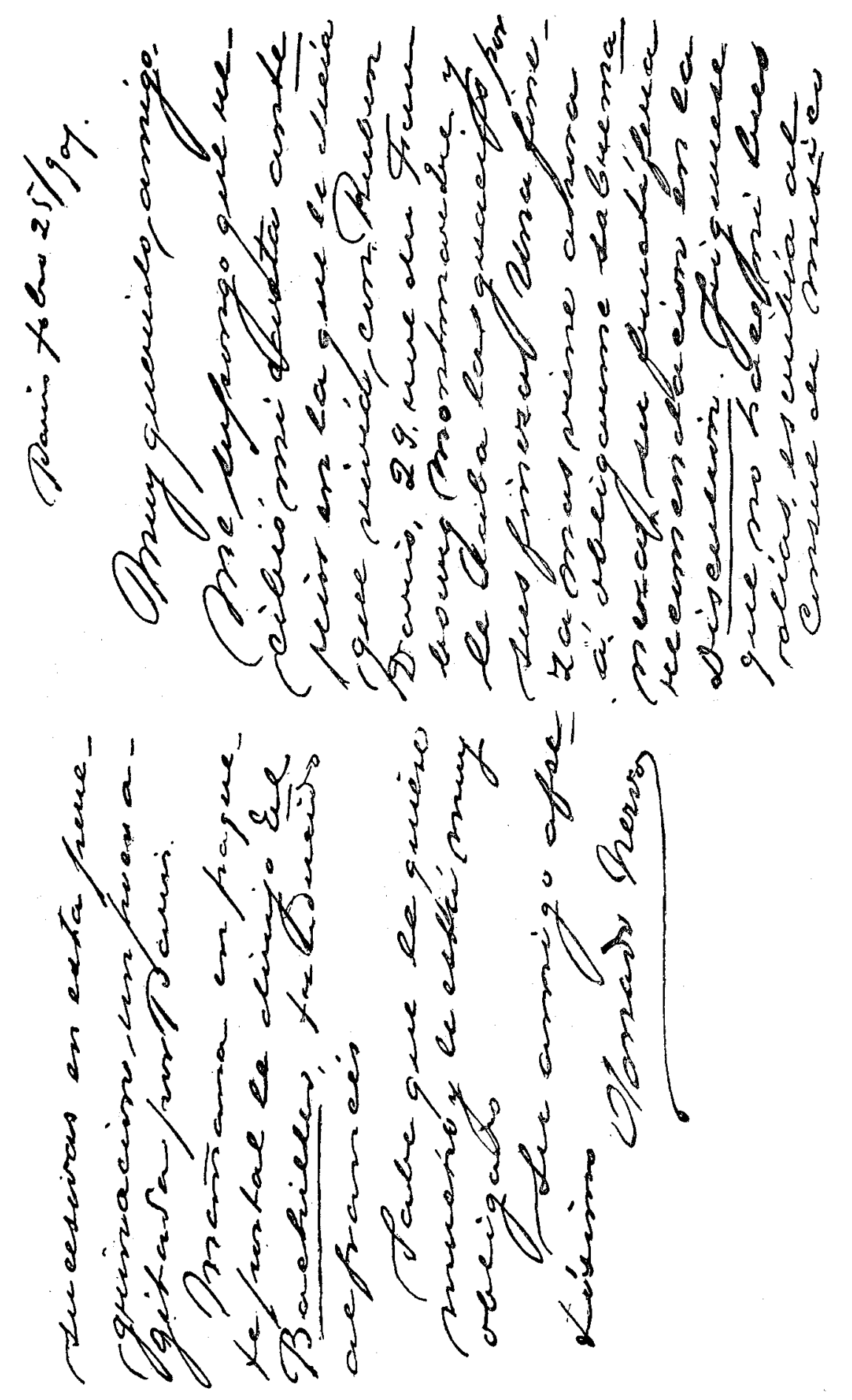




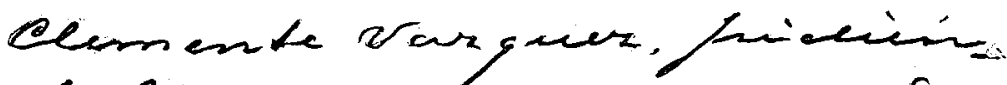

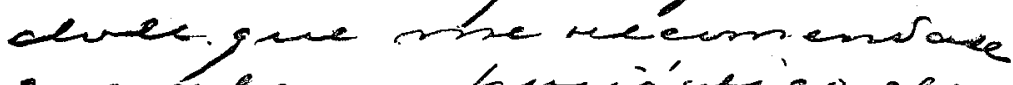

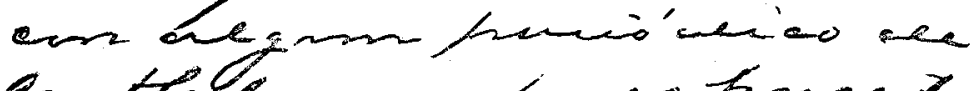

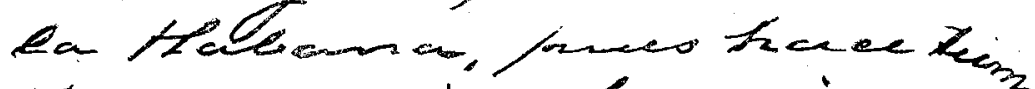

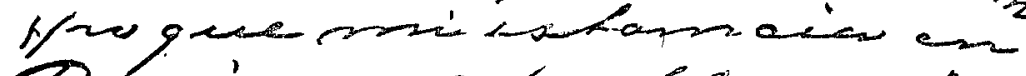
Poduis so ce proberma de le thisedecion de ens angures o'ceg. ham diftreie curb. ex.

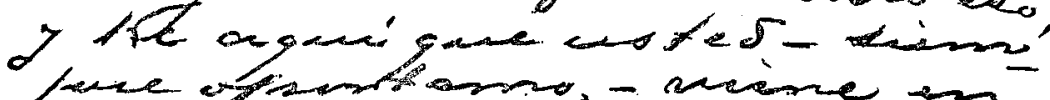

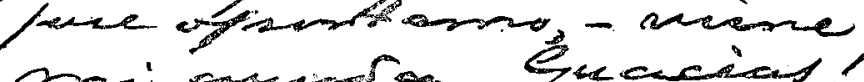

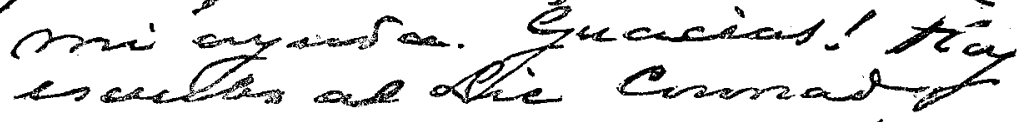

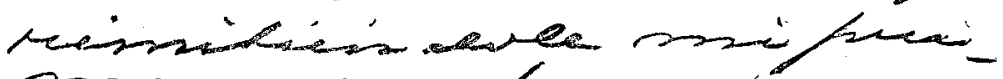
mence ementa y juck

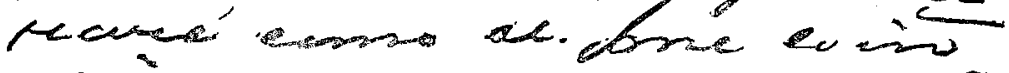

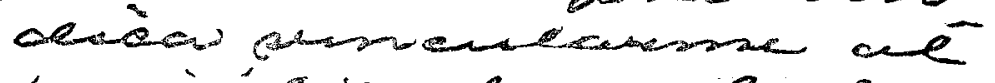
herviólever, bo cuscel wheo ole Karsins versas.

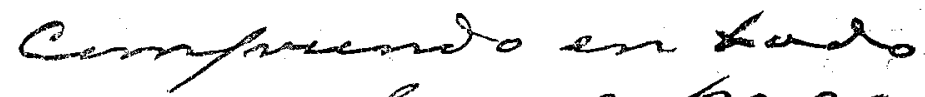
tha rowern logwe heres

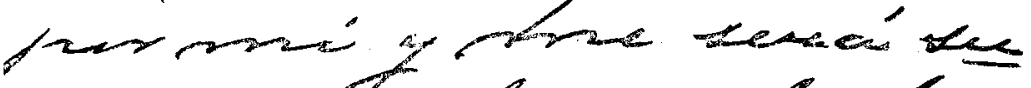

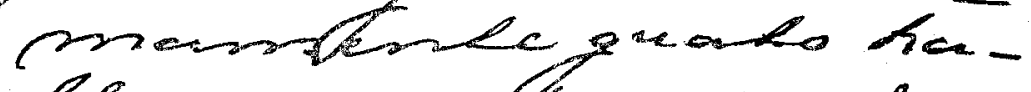
learrars a Gurmsa be

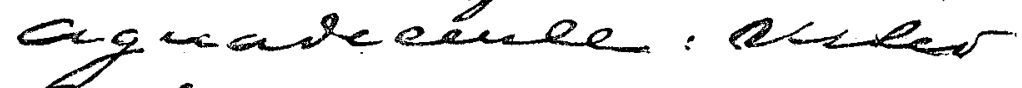
Gowe, rewan ansiert Letay.

Hanno ce ki

gthe dewares y yo as

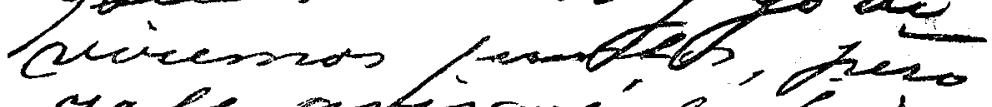

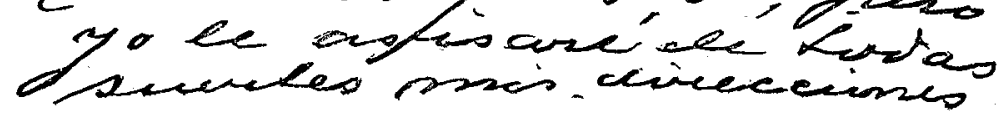




\section{Abril 30/90I}

M. le Dr. J. Mojarrieta

Hotel Metropole

Rue Castiglione

E. V.

Muy querido amigo:

Sé que está aquí y me sería grato verle. Como es posible que no coincidiésemos en la rue du Faubourg Montmartre 29, pues estas tardes salgo con frecuencia a diversos asuntos, sírvase darme un rendez-vous, en cualquier parte.

Acababa de escribirle a Cuba, ignorando su viaje.

Muy suyo

(F) Nervo 


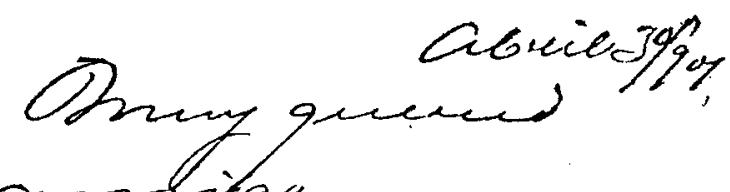

anshige.

Le lace extión an -

quí z me sucé gra

Lo muce. Cmmles pivialo gree no ean

rue dur jiandurer?

Anmteriantre 29, fues estar hardes pale con Precuencia a'laciess.

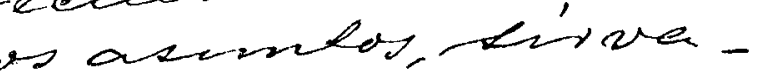
dey vars. en eccale guin ficule.

Acaterta derencule. lea' Cula, izmonam Tे twe viagle

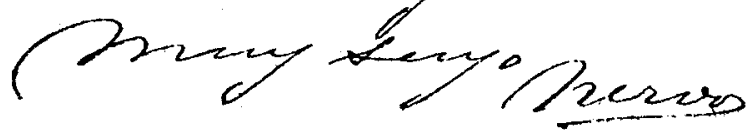


